\title{
Competitive Bidding with a Bid Floor
}

\author{
Bin Chen and Y. Stephen Chiu ${ }^{* \dagger}$
}

2 July 2009

\begin{abstract}
We study competitive bidding with an explicit bid floor, motivated by minimum wage legislation and minimum labor standard imposed from NGOs or organized labor. We derive the equilibrium strategies in, and compare the expected procurement costs among, the second-price, first-price, English, and Dutch auctions in a private-cost model. For the English auction, we also consider a variant motivated by the buy-it-now option in eBay auctions. We find that the first-price auction and the aforementioned English auction lead to exactly the same outcome, corresponding to a lower expected procurement cost than under the second-price auction and the standard English auction.
\end{abstract}

JEL Classification: C70, D44, L11.

Keywords: Competitive bidding, bid floor, minimum wage, revenue equivalence

\section{Introduction}

In this paper, we study competitive bidding with an explicitly imposed price floor. Price control is not uncommon in market economies. Anti-usury regulation dictates an upper limit to the interest rate. Though rent control is getting less popular, minimum wage legislation continues to be commonplace in all advanced industrial

\footnotetext{
${ }^{*}$ Chen: School of Economics and Finance, University of Hong Kong, Pokfulam Road, HONG KONG. Email: chinabin@hku.hk. Chiu (corresponding author): School of Economics and Finance, University of Hong Kong, Pokfulam Road, HONG KONG. Email: schiu@econ.hku.hk.

${ }^{\dagger}$ We would like to thank Francis Cheung and Rachel Yang for useful discussions. Any remaining errors are our own. Financial support from the Hong Kong Research Grants Council (HKU742407H) is gratefully acknowledged.
} 
countries. While the effect of price control is well understood in a perfectly competitive environment, this paper conducts the counterpart study in an imperfectly competitive environment, more specifically, in a competitive bidding environment in which a monopsony buyer procures from a couple of potential sellers. This exercise is motivated not simply out of curiosity; there are reasons that price control is relevant for such kind of markets.

First, imagine that a government agency is auctioning out a service contract that requires, among other things, a certain number of minimum work hours. The minimum wage legislation is thus translated into a lower bound any bid below which will look suspicious, justifying the imposition of a price floor for any bid to be deemed valid. Second, a monopsony-buyer may be thwarted from accepting very low bids because of pressure from organized labor and NGOs, who are concerned about decent work standard and non-exploitation of low wage workers: this consideration is particular acute if the bidding involves foreign suppliers from less developed countries. Third, the monopsony-buyer may also be thwarted from accepting a bid from a bidder who is engaging in predatory bidding, i.e., who has submitted a low bid with the purpose to drive out competing bidders from the market. ${ }^{1}$ So noticeable is the alleged problem of abnormally low bids that the European Union has had deliberation on their detection and prevention. Such deliberation can be viewed as instituting a price floor below which bids are disqualified. ${ }^{2}$

In this paper, we study the effect of an explicitly imposed price floor in competitive bidding through which a single buyer procures a single unit of good or service from one of several sellers. We study the equilibrium strategies as well as the expected procurement costs for the four types of standard auctions, namely the second-price, first-price, English, and Dutch auctions. In terms of the English auction, we also consider a variant in which any seller is allowed to terminate the auction by selling at the price equal to the bid floor, motivated by the Buy-It-Now option in eBay auctions. We focus ourselves on an environment of symmetric, risk neutral bidders with private costs which might be lower than the price floor. In the absence of restrictions on the bidding price, this is the environment where the same expected procurement costs will prevail among the four types of auctions (see, i.e., Myerson (1981), Riley and Samuelson (1981), McAfee and McMillan (1987)).

\footnotetext{
${ }^{1}$ In addition to predatory bidding, abnormally low bids may also result from faulty calculation, or from opportunist behavior arising from anitcipation of renegotiation of a better contract after winning by underbidding. (Alexandersson and Hulten (2006) examine each of these three reasons.)

${ }^{2}$ See DGIII Working Group on Abnormally Low Tenders (1999).
} 
As it is usually the case, a buyer is hurt by a price floor. Instead of simply confirming this idea, our result suggests that, in the context of competitive bidding, the standard auction formats that we usually consider to be equivalent are no longer equivalent and we are able to point out which format is best in avoiding the adverse effect on the monopsony buyer as far as expected procurement costs are concerned. Our main finding is that the first-price auction and the aforementioned variant of English auction lead to exactly the same outcome, corresponding to a lower expected procurement cost than under the second-price auction and the standard English auction.

The equivalence of the first-price auction and the aforementioned variant of English auction is quite intriguing. We find that a seller who will bid at the price floor in the former auction if, and only if, she will jump down to sell at a posted price at the beginning in the latter auction, leading to payoff equivalence between the two auctions for all agents. The derivation of the equilibrium strategy under that English auction is similar to the analysis of online auctions (see, e.g., Budish and Takeyama 2001, Chiu and Cheung 2004, Mathew 2004, Reynold and Wooders 2009, and Hidvegi, Wang and Whinston 2006). This paper can be alternatively viewed as one comparing the expected revenue under different forward auctions in which there is a common upper bound to bid values. Our work thus helps clarify the relationship between online auctions with the Buy-It-Now clause to first-price auctions with exogenously imposed price ceilings.

Despite few studies of competitive bidding with a bid floor, some recent papers do take notice of a bid ceiling in the forward auction. For example, Che and Gale (1998a) as well as Gavious, Moldovanu and Sela (2002) look at bid ceilings in the common value all-pay auctions, and justify the caps on contributions by political lobbies. Banerjee and Chakraborty (2005) find that bid ceiling reduces the winner' curse in the symmetric common value auctions, and hence encourages the bidders to bid more aggressively. It should be emphasized that this line of studies is concerned about common value auctions, while our framework is concerned about private value auction (when being rephrased as a forward auction).

Closest to ours are the papers by Che and Gale (1998b), Zhang (2001), and Calveras, Ganuza and Hauk (2004), who study (forward) auctions with budget constraints. These papers are all motivated by the fact that buyer-bidders may have financial constraints. Che and Gale show that the expected revenue under the firstprice auction is higher than that under the second-price auction, and the intuition is 
that financial constraints are more likely to bind in the latter form of auction. Zhang studies whether, or to what extent, financing from the auctioneer to winning bidders will improve efficiency as well as the auctioneer's payoff when bidders are financially constrained. Calveras, Ganuza and Hauk study a similar model, asking whether, or to what extent, surety bond provided from a third party may help prevent renege after the auction. Unlike in our setting where the same, commonly known price floor is applied to all bidders, in all of these papers, the financial constraints differ from bidder to bidder and are bidders' private information. This thus leads to very different characterization of the equilibrium bidding strategy in the first-price sealed bid auction.

The rest of the paper is organized as follows. Section 2 formally characterizes the equilibrium bidding strategies of the first-price and second-price auctions. Section 3 studies two kinds of English auctions. Section 4 and 5 compares and contrasts the expected procurement costs under auctions with the bid floor. Section 6 compares and contrasts the expected procurement costs under auctions with versus without the bid floor. Section 7 concludes. In this paper, all proofs are relegated in the appendix unless otherwise stated.

\section{Second-price and first-price auctions}

\subsection{Primitives}

A buyer plans to hold a competitive bidding, purchasing a single unit of good or service from one of $N \geq 2$ sellers. Each seller, identified by an index $i=1, \ldots, N$, incurs a $\operatorname{cost} c_{i}$ to produce this item. While $c_{i}$ is known to seller $i$, it is uncertain to the others. We assume that $c_{i}$ is independently and identically distributed according to an increasing cumulative function $H$, with a continuous associated density $h$, defined over the support of $[0, \bar{c}]{ }^{3} Y_{j}^{(k)}$ denotes the $j$ th lowest cost among $k$ sellers. $G^{(k)}$ and $g^{(k)}$ denote the cumulative function and density function of the first-order statistic $Y_{1}^{(k)}$. All parties involved are risk neutral.

There are four types of classical auctions for us to study, namely, the first-price auction, second-price auction, English auction, and Dutch auction. In each auction form, there is an exogenously given, commonly known price floor $\underline{b}>0$. We view this price floor as externally imposed, in the fashion like the minimum wage legislation,

\footnotetext{
${ }^{3}$ In Section 6 , we also assume that $h / H$, the reverse hazard rate, is decreasing in its argument; in other part of this paper, this assumption is not necessary.
} 
which does not necessarily reflect the interest of the auctioneer-buyer. We assume that the products provided by different sellers (i.e., bidders in our analysis) are of the same quality, and the contract awarded by the auction could be perfectly enforced without occurrence of any renegotiation. We also assume that the utility produced by the item, $B$, exceeds $\bar{c}$, and that the buyer always buys an item provided that the price is less than $B$. Therefore, the buyer's primary concern is the expected procurement cost subject to his buying an item. ${ }^{4}$

We denote the above auction games as $\Gamma_{t}$, where $t$ represents the respective auction form $F$ (first-price), $S$ (second-price), $D$ (Dutch), and $E$ (English). For $\Gamma_{t}$, let $B^{t}\left(c_{i}\right)$ be the bidding strategy of seller $i$, and $E m^{t}$ be the expected payment made by the buyer. (Since we only consider symmetric equilibrium, subscript $i$ of $B_{i}^{t}$ is omitted.) To facilitate our analysis, we define auxiliary auction $\Gamma_{\widehat{t}}$, which is the same as $\Gamma_{t}$, except that the price floor is not in place. Accordingly, we define $B^{\widehat{t}}\left(c_{i}\right)$ and $E m^{\widehat{t}}$.

\subsection{Second-price auction}

In $\Gamma_{\widehat{S}}$, where there is no price floor, each seller is able to play her weakly dominant strategy, which is to bid her true cost (we use "he" to describe the buyer and "she" to describe each seller). This indeed constitutes a symmetric equilibrium. More formally, in the equilibrium, the bidding strategy of seller $i$ is

$$
B^{\widehat{S}}\left(c_{i}\right)=c_{i}, \quad \forall c_{i} \in[0, \bar{c}] .
$$

It is straightforward to verify that, in the presence of the price floor, each seller would adopt the same strategy except that, in case her cost is below the price floor, she submits a bid exactly equal to the price floor.

Proposition 1 Consider the second-price auction $\Gamma_{S}$, in which there is a price floor $\underline{b}>0$. The equilibrium bidding strategy of the seller who has a cost of $c_{i}$ is as follows

$$
B^{S}\left(c_{i}\right)=\left\{\begin{array}{cll}
\underline{b}, & \text { if } & c_{i} \leq \underline{b} \\
B^{\widehat{S}}\left(c_{i}\right), & \text { if } & c_{i}>\underline{b} .
\end{array}\right.
$$

\footnotetext{
${ }^{4}$ It is well known in the forward auction literature that the optimal forward auction would involve setting a reserve price below which the monopoly-seller will not sell her good. Thus in the context of reverse auction, the optimal auction will involve setting a reserve price above which the monopsony-buyer will not buy. It is important to notice that, even if $B>\underline{c}$, the reserve price may well be smaller than $\underline{c}$. In this paper, however, we do not study reserve price.
} 


\section{Proof. Omitted.}

\subsection{First-price auction}

To study the first-price auction with the price floor, $\Gamma_{F}$, we first examine $\Gamma_{\widehat{F}}$, the counterpart auction without the price floor. In the equilibrium of $\Gamma_{\widehat{F}}$, each seller's bid is an increasing function of her own cost $c_{i}$, and is given by: $\forall c_{i} \in[0, \bar{c}]$,

$$
\begin{aligned}
B^{\widehat{F}}\left(c_{i}\right) & =c_{i}+\frac{\int_{c_{i}}^{\bar{c}}(1-H(t))^{N-1} d t}{\left(1-H\left(c_{i}\right)\right)^{N-1}} \\
& =E\left[Y_{1} \mid Y_{1}>c_{i}\right]
\end{aligned}
$$

where $Y_{1}=Y_{1}^{(N-1)}$ (the superscript $(N-1)$ is omitted when no confusion is caused). The two expressions in the two lines (1) and (2) are equivalent, but one may be easier to use than the other under some circumstances. Because the seller's equilibrium bid exceeds her cost, it is possible that $B^{\widehat{F}}\left(c_{i}\right)$ still exceeds $\underline{b}$ even though $c_{i}$ does not. Thus, there are two cases to consider. Referring to Figure 1, Panel a depicts the case where the bidding function never takes on a value below $\underline{b}$; Panel b depicts the other case where it may. Of course, the difference between the two panels is due to the fact that the price floor is low enough in Panel a, but is sufficiently high in Panel b.

Now let us come back to $\Gamma_{F}$, where the price floor is in place. We first notice that in case $B^{\widehat{F}}(0) \geq \underline{b}$, as the price floor is not binding, $B^{\widehat{F}}$ is indeed an equilibrium bidding strategy in $\Gamma_{F}$. However, in case $B^{\widehat{F}}(0)<\underline{b}$, the price floor is binding, $B^{\widehat{F}}$ cannot be an equilibrium bidding strategy in $\Gamma_{F}$. One may speculate that, $B^{F}$ is just a truncation of $B^{\widehat{F}}$ at $c_{0}$, that is, $B^{F}\left(c_{i}\right)=\underline{b}$ for $c_{i}<c_{0}$ and $B^{F}\left(c_{i}\right)=B^{\widehat{F}}\left(c_{i}\right)$ for $c_{i} \geq c_{0}$, where $c_{0}$ satisfies $B^{\widehat{F}}\left(c_{0}\right)=\underline{b}$. This is not correct though. In fact, any seller whose cost, $c_{i}$, slightly exceeds $c_{0}$ would prefer bidding $\underline{b}$ to bidding $B^{\widehat{F}}\left(c_{i}\right)$. By doing so, her gain conditional on winning is slightly reduced; but her winning chance increases by a discrete amount..$^{5}$ This suggests a discontinuity of the bidding function at some $c^{*}>c_{0}$ such that, with cost strictly lower than $c^{*}$, the seller bids

\footnotetext{
${ }^{5} \mathrm{We}$ illustrate this point in a two-seller case. Suppose a seller has a cost of $\left(c_{0}+\varepsilon\right)$ where $\varepsilon$ is a small positive number. By bidding $B^{\widehat{F}}\left(c_{0}+\varepsilon\right)$, her expected payoff is $\left(1-H\left(c_{0}+\varepsilon\right)\right)\left[B^{\widehat{F}}\left(c_{0}+\varepsilon\right)-\left(c_{0}+\varepsilon\right)\right]$. By bidding $\underline{b}$, her expected payoff is $\left[\left(1-H\left(c_{0}\right)\right)+(1 / 2) H\left(c_{0}\right)\right]\left[\underline{b}-\left(c_{0}+\varepsilon\right)\right]$. As $\epsilon \rightarrow 0$, the latter payoff strictly exceeds the former one.
} 


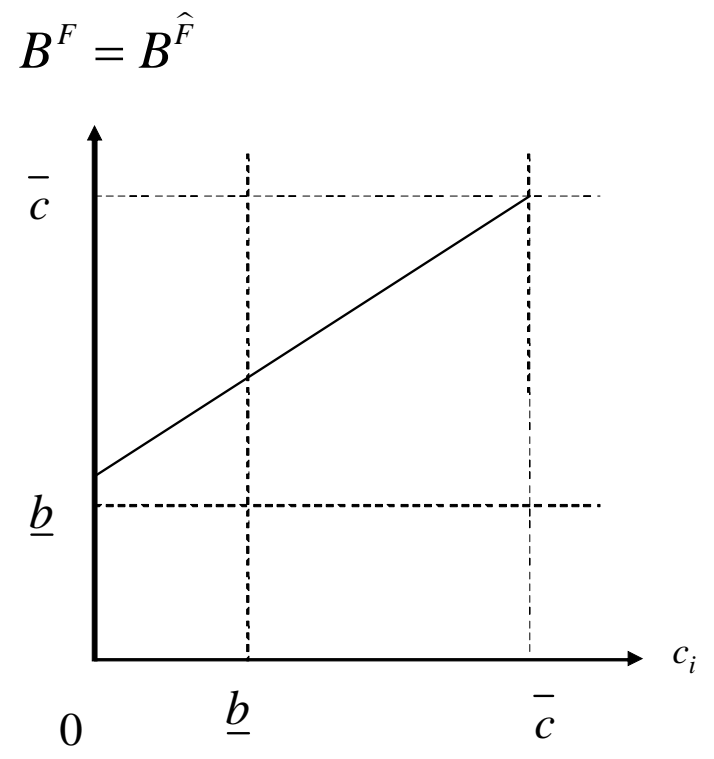

Panel a
$B^{F}$ : dashed line

$B^{\widehat{F}}$ : solid line

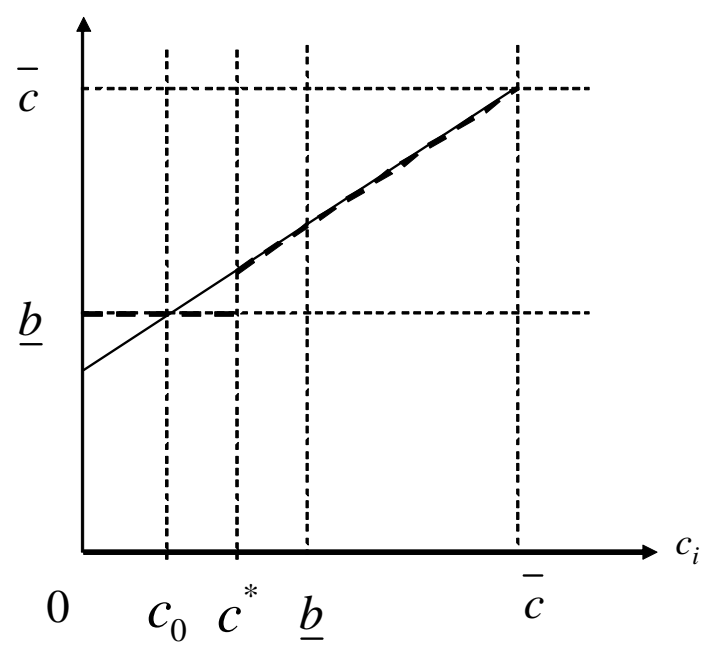

Panel b

Figure 1: In Panel a, $\underline{b}$ is small, and the equilibrium bidding function in $\Gamma_{F}$ coincides with that in $\Gamma_{\widehat{F}}$. In Panel $b, \underline{b}$ is sufficiently large, and the equilibrium bidding function in $\Gamma_{F}$ is different from that in $\Gamma_{\widehat{F}}$. 
$\underline{b}$; when the cost is greater than or equal to $c^{*}$, the seller bids according to $B^{\widehat{F}} .^{6}$ Formally, seller $i$ bids

$$
B^{F}\left(c_{i}\right)=\left\{\begin{array}{ccc}
B^{\widehat{F}}\left(c_{i}\right), & \text { if } & c_{i} \geq c^{*} \\
\underline{b}, & \text { if } & c_{i}<c^{*}
\end{array}\right.
$$

To further elaborate the above bidding function, we consider the seller whose cost exactly equals $c^{*}$. By bidding $B^{\widehat{F}}\left(c^{*}\right)$, she gains a net benefit of $B^{\widehat{F}}\left(c^{*}\right)-c^{*}$ conditional on winning and the winning probability is $\operatorname{Pr}\left(Y_{1}>c^{*}\right)$; by bidding $\underline{b}$, she gains a net benefit of $\underline{b}-c^{*}$ conditional on winning and the winning probability ${ }^{7}$ is

$$
\Omega\left(c^{*}\right)=\operatorname{Pr}\left(Y_{1}>c^{*}\right)+\sum_{j=1}^{N-1} \frac{\operatorname{Pr}\left(Y_{j}<c^{*}<Y_{j+1}\right)}{j+1} .
$$

She is indifferent between these two options when

$$
\left(B^{\widehat{F}}\left(c^{*}\right)-c^{*}\right) \times \operatorname{Pr}\left(Y_{1}>c^{*}\right)=\left(\underline{b}-c^{*}\right) \times \Omega\left(c^{*}\right) .
$$

In this case, the greater net benefit conditional on winning in the first option (i.e., bidding $\left.B^{\widehat{F}}\left(c^{*}\right)\right)$ is exactly offset by the greater winning probability in the second option (i.e., bidding $\underline{b}$ ). The following lemma ensures the existence of $c^{*}$.

Lemma 1 If $B^{\widehat{F}}(0)<\underline{b}$, there exists $c^{*} \in\left(c_{0}, \underline{b}\right)$ which satisfies $(5)$.

Proof. Motivated by (5), we define two continuous functions $L H S(c)=\left(B^{\widehat{F}}(c)-c\right) \times$ $\operatorname{Pr}\left(Y_{1}>c\right)$ and $R H S(c)=(\underline{b}-c) \times Q(c)$. We reckon that $R H S\left(c_{0}\right)>L H S\left(c_{0}\right)>$ 0 and $L H S(\underline{b})>R H S(\underline{b})=0$, implying that $R H S\left(c_{0}\right)-L H S\left(c_{0}\right)>0$ and $R H S(\underline{b})-L H S(\underline{b})<0$. According to the intermediate value theorem, there should exist some $c^{*} \in\left(c_{0}, \underline{b}\right)$ such that $R H S\left(c^{*}\right)-L H S\left(c^{*}\right)=0$, i.e., (5) is satisfied.

Now that $B^{F}$ is well defined, it is routine to verify that it is indeed an equilibrium strategy. We summarize the results as follows, and the proof is relegated to the Appendix.

\footnotetext{
${ }^{6}$ In fact, with the cost equal to $c^{*}$, the bidder is indifferent between bidding according to $B^{\widehat{F}}$ and at $\underline{b}$. It is without loss of generality to assume that in this case she bids according to $B^{\widehat{F}}$.

${ }^{7}$ By bidding $\underline{b}$, she wins either if all other sellers have costs strictly exceed $c^{*}$ or if at least one rival seller has a cost lower than or equal to $c^{*}$. In the former case, she wins with probability one; in the latter case, she ties with those bidders. (4) takes into account both circumstances.
} 
Proposition 2 Consider the first-price auction $\Gamma_{F}$, in which there is a price floor $\underline{b}>0$.

(a) If $B^{\widehat{F}}(0) \geq \underline{b}$, there exists an equilibrium in which all sellers submit bids equal to $B^{\widehat{F}}$.

(b) If $B^{\widehat{F}}(0)<\underline{b}$, there exists an equilibrium in which all sellers use $B^{F}$ as defined in (3).

The pioneering work by Che and Gale (1998b), as well as Calveras, Ganuza and Hauk (2004) and Zhang (2001), studies the forward auction where bidders are financially constrained, which can be viewed as the counterpart of the reverse auction with a bid floor. A subtle difference there is that each bidder's financial constraint is her private information. Given that each such constraint is described by a nicely behaved cumulative distribution function, there is no discontinuity in the equilibrium bidding strategy. On the other hand, in the present paper, the price floor constraint is identical, commonly known, and applying to all bidders. This thus results in a discontinuity in their bidding strategy.

\section{English auction}

We study two kinds of English auctions with bid floors. In the first kind, denoted by $\Gamma_{E A}$, the bid drops continuously until there remains only one active seller, who then becomes the winner ${ }^{8}$ in case of a tie at a price of $\underline{b}$, a random draw will be made among the remaining active sellers. Clearly, there exists a symmetric equilibrium in which each seller remains active until the price drops to her private cost or $\underline{b}$, whichever is higher. It is obviously that this outcome is revenue equivalent to the symmetric equilibrium studied in the second-price auction, and in this sense the analysis is completed.

What is more interesting is another kind of English auction, in which any seller may jump down to the price of $\underline{b}$, at the outset or in the middle of the auction process. ${ }^{9}$ By adopting such an act, the auction is terminated as the contract is awarded to the seller, or any other seller who has also jumped down at the same moment. In what follows, we focus on this type of English auction, and denote it by

\footnotetext{
${ }^{8}$ The English auction executed through continuous movement of the bid price is also known as the Japanese English auction.

${ }^{9}$ We do not allow more general kind of bid jumps, which play an subtle role in English auction with affiliated values, but not in English auction with private values. See Avery (1998) on this.
} 
$\Gamma_{E B}$. Analytically, we assume that, despite knowing the initial number of sellers $N$, after the auction starts, no active bidders know who and how many sellers have left the auction. What we have in mind is that the auction is held online.

In $\Gamma_{E B}$, for a seller with a cost exceeding $\underline{b}$, she will simply stay active until the price drops to her own cost, and never jump down. For a seller with a cost below $\underline{b}$, because even selling at the price of $\underline{b}$ brings in some profits, she certainly will preempt knowing that somebody may jump down in the next moment. What it means is that her strategy is to decide on the exact moment, i.e., prevailing price, at which she will jump down; we denote this price as a function of the seller's cost, $\beta\left(c_{i}\right)$, where $c_{i} \in[0, \underline{b})$. Intuitively, this function is decreasing is $c_{i}$; that is, the lower $c_{i}$ is, the earlier seller $i$ is to jump down. It also implies that, if the costs of some sellers are sufficiently low, they may use jump-down strategy at the outset of the auction. Thus, there are indeed two cases for us to consider, as depicted in Figure 2.

Referring to Figure 2, in Panel a, the jump-down strategy is depicted by a downward slopping curve, with $\beta\left(c_{i}\right) \in[\underline{b}, \bar{c})$ for all $c_{i} \in[0, \underline{b})$, and no sellers immediately jump down at the outset of the auction; in this case, the equilibrium is efficient, since the eventual winner turns out to be the least costly seller. In Panel b, the sellers with costs less than $c^{* *}$ would jump down at the outset of the auction, and the sellers whose costs fall into the interval of $\left(c^{* *}, \underline{b}\right)$ would wait to jump down according to $\beta$; in this case, the equilibrium is inefficient, because all sellers with costs less than $c^{* *}$ have the equal chance of winning. Obviously, Panel a is relevant when $\underline{b}$ is low enough, and Panel $\mathrm{b}$ is relevant otherwise.

Furthermore, as will be shown, the jump-down function $\beta\left(c_{i}\right)$ satisfies a simple relationship:

$$
E\left[Y_{1} \mid c_{i}<Y_{1}<\beta\left(c_{i}\right)\right]=\underline{b} .
$$

In case that $\beta(0)$ solved from (6) has a solution, the jump-down strategy of any seller $i$ with $\operatorname{cost} c_{i}<\underline{b}$ is completely characterized by $\beta\left(c_{i}\right)$. In case that $\beta(0)$ solved from (6) does not have a solution, the jump-down strategy of any seller $i$ with cost $c_{i}<\underline{b}$ is represented by

$$
B^{E B}\left(c_{i}\right)=\left\{\begin{array}{clc}
\beta\left(c_{i}\right), & \text { if } & c^{* *} \leq c_{i}<\underline{b} \\
\bar{c}, & \text { if } & c_{i}<c^{* *}
\end{array}\right.
$$

We further elaborate the choice of the seller with a cost of $c^{* *}$. By waiting to 
When to jump down

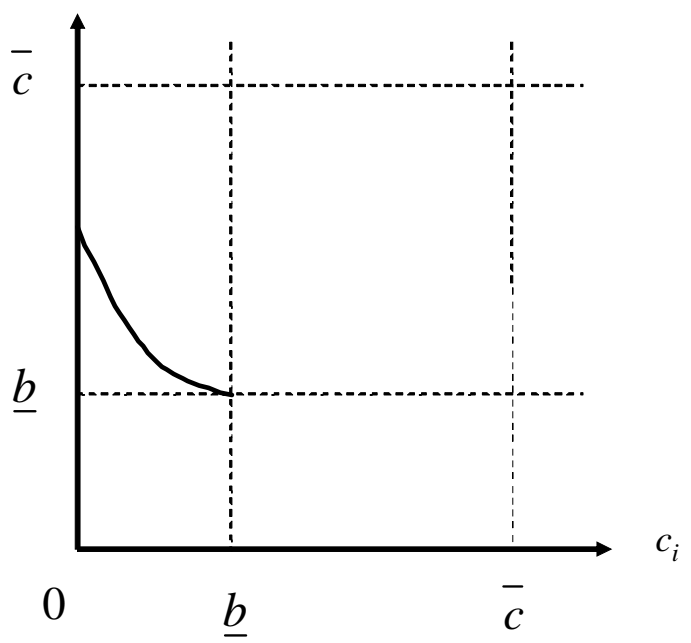

Panel a
When to jump down

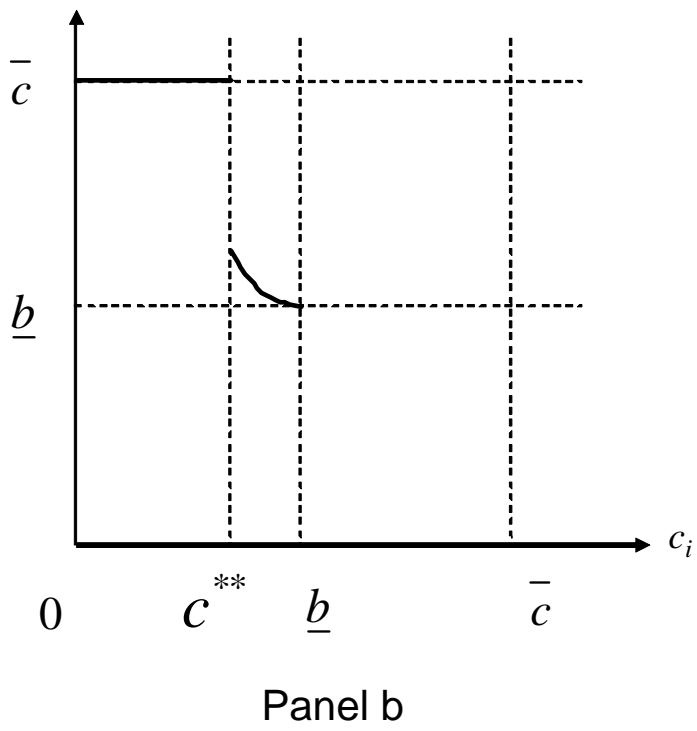

Figure 2: In Panel a, $\underline{b}$ is small, and no sellers will jump down at the outset of the auction, $\Gamma_{E B}$; in Panel b, $\underline{b}$ is sufficiently large, and sellers may jump down at the outset. 
jump down until the price drops to $\beta\left(c^{* *}\right)$, she wins the auction, either if (i) all other sellers have costs greater than $\beta\left(c^{* *}\right)$ (with probability $\operatorname{Pr}\left(Y_{1}>\beta\left(c^{* *}\right)\right)$ ), or if (ii) nobody has jumped down earlier (with probability $\operatorname{Pr}\left(c^{* *}<Y_{1}<\beta\left(c^{* *}\right)\right)$ ). In scenario (i), she receives an expected payment of $E\left[Y_{1} \mid Y_{1}>\beta\left(c^{* *}\right)\right]$; in scenario (ii), she receives a payment of $\underline{b}$. However, by bidding $\underline{b}$ at the outset of the auction, she wins with a greater probability, represented by $\Omega\left(c^{* *}\right)$ (which is defined in the same formula as (4) except for $c^{*}$ being replaced by $c^{* *}$ ), and conditional on winning she receives a payment of $\underline{b}$. Thus, the seller with a cost equal to $c^{* *}$ is indifferent between these two options, when

$\left(E\left[Y_{1} \mid Y_{1}>\beta\left(c^{* *}\right)\right]-c^{* *}\right) \times \operatorname{Pr}\left(Y_{1}>\beta\left(c^{* *}\right)\right)+\left(\underline{b}-c^{* *}\right) \times \operatorname{Pr}\left(c^{* *}<Y_{1}<\beta\left(c^{* *}\right)\right)=\left(\underline{b}-c^{* *}\right) \times \Omega\left(c^{* *}\right)$.

Given that $c^{* *}$ is defined by (8), the discontinuity of the jump-down strategy in the above formula follows the same argument for the discontinuity of equilibrium strategy in $\Gamma_{F}$. The following proposition summarizes the equilibrium strategy in $\Gamma_{E B}$.

Proposition 3 Consider the English auction $\Gamma_{E B}$, where bids lower than $\underline{b}$ are forbidden and jump-down strategy is allowed.

(a) If $\beta(0)<\bar{c}$, there exists an equilibrium in which each seller with cost $c_{i} \in[\underline{b}, \bar{c}]$ stays active until the price drops to $c_{i}$; each seller with cost $c_{i} \in[0, \underline{b})$ will adopt jump-down strategy $\beta$, as described in (6).

(b) If $\beta(0) \geq \bar{c}$, there exists an equilibrium in which each seller with cost $c_{i} \in[\underline{b}, \bar{c}]$ stays active until price drops to $c_{i}$; each seller with cost $c_{i} \in[0, \underline{b})$ will adopt jumpdown strategy $B^{E B}$, as described in (7).

The reader may find this auction format familiar; the jump-down feature is essentially the same as eBay's Buy-It-Now option which has gained attention recently in the literature. The equilibrium strategy for eBay auctions has been independently derived by several authors (see Budish and Takeyama 2001; Chiu and Cheung 2004; Matthew 2004; Reynold and Wooders 2009; Hidvegi, Wang and Whinston 2006; just to list a few). More details about the derivation of the strategy for the current game can be found in the Appendix. Amazon and Yahoo! have a different type of posted price options, which is available only before the auction takes place (or "at the outset" in the language of this paper); once the auction has started, nobody can 
exercise the posted price option anymore. (See Chiu and Cheung for a comparison and contrast. $)^{10}$

\section{Expected procurement costs: first-price vs second- price auctions}

Now that we have obtained the equilibrium bidding strategies in the first-price and second-price auctions, we are ready to show that the expected cost is strictly lower under the first-price auction than under the second-price auction. Suppose $\underline{b}$ is low enough so that $B^{\widehat{F}}(0) \geq \underline{b}$. In this case, in the first-price auction, the price floor is non-binding, and hence it follows that the expected procurement cost remains unchanged before and after we introduce this constraint, i.e., $E m^{F}=E m^{\widehat{F}}$. On the other hand, the price floor is binding in the second-price auction. A seller who otherwise would submit a bid below $\underline{b}$ in $\Gamma_{\widehat{S}}$ is restricted to bidding $\underline{b}$ in $\Gamma_{S}$. It thus follows that the expected procurement cost in the presence of the price floor is strictly higher than that in its absence. As a result, we have $E m^{S}>E m^{F}$ in the case where $B^{\widehat{F}}(0) \geq \underline{b}$.

The other case, where $B^{\widehat{F}}(0)<\underline{b}$, is more complicated, and we illustrate it with a two-seller case. In figure 3 , every point in the $\left(c_{1}, c_{2}\right)$-space represents a realization of the costs of the two sellers. We partition the space into four different regions: A, B, $\mathrm{C}$, and $\mathrm{D}$, representing the sub-space of $\left[0, c^{*}\right) \times\left[0, c^{*}\right),\left[0, c^{*}\right) \times\left[c^{*}, \bar{c}\right],\left[c^{*}, \bar{c}\right] \times\left[0, c^{*}\right)$, and $\left[c^{*}, \bar{c}\right] \times\left[c^{*}, \bar{c}\right]$, respectively. Notice that, conditional on Region A being reached, the expected procurement costs are the same under the first-price and second-price auctions; in both auctions, both the lowest bid and second lowest bid are $\underline{b}$, and the winner is paid a price of $\underline{b}$. We next notice that, conditional on $\mathrm{B}$, and likewise $\mathrm{C}$, being reached, the expected procurement cost under the first-price auction is simply $\underline{b}$ (because the winner has bidden $\underline{b}$ ) and the corresponding cost under second-price auction is strictly greater than $\underline{b}$ (because the second highest bid may exceed $\underline{b}$ ). Finally, we notice that, conditional on D being reached, the expected procurement cost under the first-price auction is strictly lower than that under the second-price auction. The reason is that, conditional on D being reached, the bidding strategy under $\Gamma_{F}$ is the same as that under $\Gamma_{\widehat{F}}$ (because the price floor is non-binding), while the bidding strategy under $\Gamma_{S}$ is greater than that under $\Gamma_{\widehat{S}}$ (because the price floor

\footnotetext{
${ }^{10}$ The online auctions like as those found eBay have other features not found in our model. See, for instance, Roth, A., and A. Ockenfels (2002), on last minute bidding and how the bidding ends.
} 


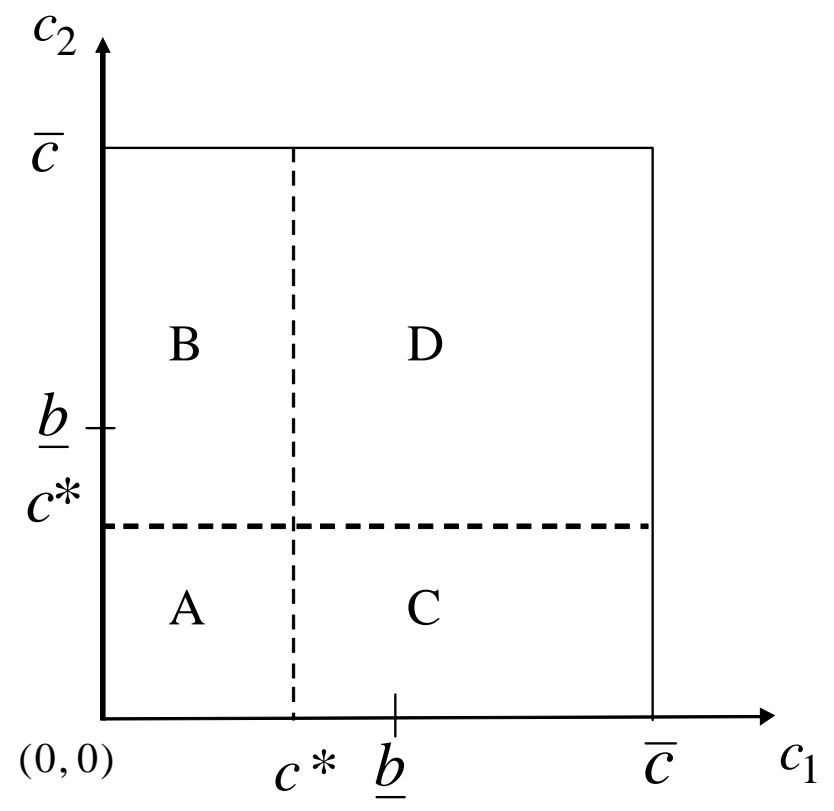

Figure 3: A partition of the type space $\left(c_{1}, c_{2}\right)$ for two-bidder case assuming $c^{*}>0$ exists. 
is now binding for some range in Region D). Thus, all these taken together suggest that the expected procurement cost under the first-price auction, $E m^{F}$, is strictly lower than that under the second-price auction, $E m^{S}$, in the case where $B^{\widehat{F}}(0)<\underline{b}$.

We state the general result (where there may be more than two bidders) as follows.

Proposition 4 Suppose that no bids below $\underline{b}$ are allowed. The expected procurement cost is strictly lower under the first-price auction than that under the second-price auction, i.e., $E m^{F}<E m^{S}$.

Proof. By definition, the expected procurement costs under $\Gamma_{F}$ and $\Gamma_{S}$ are, respectively,

$$
\begin{aligned}
E m^{F}= & \int_{\mathbf{c} \notin\left[c^{*}, \bar{c}\right]^{N}} \min \left\{B^{F}\left(c_{1}\right), \ldots, B^{F}\left(c_{N}\right)\right\} d \mathbf{H}(\mathbf{c}) \\
& +\int_{\mathbf{c} \in\left[c^{*}, \bar{c}\right]^{N}} \min \left\{B^{F}\left(c_{1}\right), \ldots, B^{F}\left(c_{N}\right)\right\} d \mathbf{H}(\mathbf{c}) \\
E m^{S}= & \int_{\mathbf{c} \notin\left[c^{*}, \bar{c}\right]^{N}} \sec \min \left\{B^{S}\left(c_{1}\right), \ldots, B^{S}\left(c_{N}\right)\right\} d \mathbf{H}(\mathbf{c}) \\
& +\int_{\mathbf{c} \in\left[c^{*}, \bar{c}\right]^{N}} \sec \min \left\{B^{S}\left(c_{1}\right), \ldots, B^{S}\left(c_{N}\right)\right\} d \mathbf{H}(\mathbf{c}),
\end{aligned}
$$

where $\mathbf{H}($.$) is the cumulative distribution function of \mathbf{c}=\left(c_{1}, \ldots, c_{N}\right)$ and sec min means "selecting the second lowest value among the set."

Next notice that the integrand inside the first term in the RHS of (9) is $\underline{b}$ because when $\mathbf{c} \notin\left[c^{*}, \bar{c}\right]^{N}$ at least one seller submits a bid equal to $\underline{b}$, and that the integrand inside the first term in the RHS of (10) exceeds $\underline{b}$ (provided that $c^{*}>\underline{c}$ ) because the second least costly seller may bid greater than $\underline{b}$. Therefore, to show the claim, it suffices to show the second terms in the RHSs of (9) and (10).

Define an auxiliary cumulative distribution function $D$ over $\left[c^{*}, \bar{c}\right]$, with associated density function $d$, where

$$
D\left(c_{i}\right)=\frac{H\left(c_{i}\right)-H\left(c^{*}\right)}{1-H\left(c^{*}\right)},
$$

with $D\left(c^{*}\right)=0, D(\bar{c})=1$, and $d\left(c_{i}\right)=h\left(c_{i}\right) /\left(1-H\left(c^{*}\right)\right)$. $D$ is nothing but the cumulative distribution derived from $H$ conditional on $c_{i} \in\left[c^{*}, \bar{c}\right]$. The second term 
in the RHS of (9) can be rewritten as

$$
\left(1-H\left(c^{*}\right)\right)^{N} \times \int_{\mathbf{c} \in\left[c^{*}, \bar{c}\right]^{N}} \min _{i}\left\{c_{i}+\frac{\int_{c_{i}}^{\bar{c}}(1-D(t))^{N-1} d t}{\left(1-D\left(c_{i}\right)\right)^{N-1}}\right\} d \mathbf{D}(\mathbf{c}),
$$

where $\mathbf{D}($.$) is the cumulative distribution function of \mathbf{c}=\left(c_{1}, \ldots, c_{N}\right)$, derived from $D$. Likewise, the second term in the RHS of (10) can be rewritten as

$$
\begin{aligned}
& \left(1-H\left(c^{*}\right)\right)^{N} \times \int_{\mathbf{c} \in\left[c^{*}, \bar{c}\right]^{N}} \sec \min \left\{B^{S}\left(c_{1}\right), \ldots, B^{S}\left(c_{N}\right)\right\} d \mathbf{D}(\mathbf{c}) \\
> & \left(1-H\left(c^{*}\right)\right)^{N} \times \int_{\mathbf{c} \in\left[c^{*}, \bar{c}\right]^{N}} \sec \min \left\{c_{1}, \ldots, c_{N}\right\} d \mathbf{D}(\mathbf{c}) .
\end{aligned}
$$

The inequality is due to the fact that $B^{S}\left(c_{i}\right)=0>c_{i}$ for $c_{i} \in\left[c^{*}, \underline{b}\right)$. Therefore, to show the claim, it suffices to show that $(11)=(12)$, equivalently,

$\int_{\mathbf{c} \in\left[c^{*}, \bar{c}\right]^{N}} \min _{i}\left\{c_{i}+\frac{\int_{c_{i}}^{\bar{c}}(1-D(t))^{N-1} d t}{\left(1-D\left(c_{i}\right)\right)^{N-1}}\right\} d \mathbf{D}(\mathbf{c})=\int_{\mathbf{c} \in\left[c^{*}, \bar{c}\right]^{N}} \sec \min \left\{c_{1}, \ldots, c_{N}\right\} d \mathbf{D}(\mathbf{c})$.

Notice that the left hand side (LHS) and the RHS terms are, respectively, the expected procurement costs under the first-price and second-price auctions where bids lower than $\underline{b}$ are allowed and costs are distributed according to $\mathbf{D}$ in $\left[c^{*}, \bar{c}\right]$. By the revenue equivalence theorem the two must be equal. Therefore, the claim is shown.

To end this section, we provide two numerical examples, in which $\underline{b}$ is moderately and excessively large, respectively. In either case, the expected procurement cost is lower under the first-price auction than that under the second-price auction.

Example 1 There are two sellers. Each one's cost is i.i.d. from the uniform distribution between $[0,1]$ and the price floor is $\underline{b}=1 / 3$. In $\Gamma_{F}, B^{F}\left(c_{i}\right)=\left(1+c_{i}\right) / 2$ and the price floor is never binding and $E m^{F}=E m^{\widehat{F}}=0.667$. On the other hand, $E m^{S}=0.679$.

Example 2 There are two sellers. Each one's cost is i.i.d. from the uniform distribution between $[0,1]$ and the price floor is $\underline{b}=3 / 5$. In $\Gamma_{F}, B^{F}\left(c_{i}\right)=\left(1+c_{i}\right) / 2$ if $c_{i} \geq 1 / 3$ and $B^{F}\left(c_{i}\right)=3 / 5$ otherwise, and $E m^{F}=0.679<E m^{\widehat{F}}=0.667$. On the other hand, $E m^{S}=0.739$. 


\section{$5 \quad$ Expected procurement costs: English auctions}

In this section, we calculate the expected procurement costs under the two kinds of English auctions, namely $E m^{E A}$ and $E m^{E B}$, and compare them with those under the first-price and second-price auctions. Under $\Gamma_{E A}$, where jumping down is not allowed, the equilibrium strategy is exactly the same as that under the second-price auction $\Gamma_{S}$. Hence, making use of Proposition 4, we have a clear-cut result that $E m^{E A}=E m^{S}>E m^{F}$.

For $E m^{E B}$, where jumping down is allowed, the problem is more intriguing. The point is that this type of English auction yields the same expected procurement cost as the first-price auction. The key to understanding is that the cutoff cost $c^{*}$, defined in (5), turns out to equal $c^{* *}$, defined in (8).

Lemma 2 (5) and (8) are equivalent, and $c^{*}=c^{* *}$.

Proof. To prove this lemma, it suffices to show that (5) and (8) have the same solutions. Because the two equations have the same functional form in their RHSs, what remains is to show the equivalence of their LHSs. The LHS of (5) equals

$$
B^{\widehat{F}}(c) \times \operatorname{Pr}\left(Y_{1}>c\right)-c \times \operatorname{Pr}\left(Y_{1}>c\right),
$$

while the LHS of (8), with some simplification, equals

$$
\left(E\left[Y_{1} \mid Y_{1}>\beta(c)\right]\right) \times \operatorname{Pr}\left(Y_{1}>\beta(c)\right)+\underline{b} \times \operatorname{Pr}\left(\beta(c)>Y_{1}>c\right)-c \times \operatorname{Pr}\left(Y_{1}>c\right) .
$$

We now show that the first term of (13) is equal to the first two terms of (14). The 
first term of (13) equals

$$
\begin{aligned}
& B^{\widehat{F}}(c) \times \operatorname{Pr}\left(Y_{1}>c\right) \\
= & \int_{c}^{\bar{c}} y \frac{g(y)}{1-G(c)} d y \times(1-G(c)) \\
= & \int_{c}^{\beta(c)} y g(y) d y+\int_{\beta(c)}^{\bar{c}} y g(y) d y \\
= & \int_{c}^{\beta(c)} \frac{y g(y)}{G(\beta(c))-G(c)} d y \times(G(\beta(c))-G(c)) \\
& +\int_{\beta(c)}^{\bar{c}} \frac{y g(y)}{1-G(\beta(c))} d y \times(1-G(\beta(c))) \\
= & E\left[Y_{1} \mid c<Y_{1}<\beta(c)\right] \times \operatorname{Pr}\left(c<Y_{1}<\beta(c)\right) \\
& +E\left[Y_{1} \mid Y_{1}>\beta(c)\right] \times \operatorname{Pr}\left(Y_{1}>\beta(c)\right),
\end{aligned}
$$

which, because $E\left[Y_{1} \mid c<Y_{1}<\beta(c)\right]=\underline{b}$ (due to (6)), is equal to the first two terms of (14).

Lemma 2 is key to understanding the expected procurement cost equivalence between $\Gamma_{F}$ and $\Gamma_{E B}$. Here we illustrate it using the two-seller example in Figure 3. For any $\left(c_{1}, c_{2}\right)$ not in Region $\mathrm{D}$, the winner in the first-price auction $\Gamma_{F}$ is paid a price of $\underline{b}$ (which is what she asked for) and the winner in the English auction $\Gamma_{E B}$, who has jumped down at the outset, is also paid a price of $\underline{b}$. Thus, to compare the expected procurement costs, it suffices to focus on the expected procurement costs conditional on Region D being reached. Notice that, in $\Gamma_{E B}$, the seller with a cost below $\underline{b}$ will jump down in the middle of the auction. As the seller with a smaller cost jumps down before the seller with a higher cost, the winner is always the least costly seller. Therefore, both the first-price auction $\Gamma_{F}$ and the English auction $\Gamma_{E B}$ exhibit the same two characteristics: (i) the auctions are efficient with the winner being the least costly seller; (ii) any seller with the highest possible cost, $\bar{c}$, is expected to receive a payment of zero. By the revenue equivalence theorem, these two conditions ensure the same expected procurement costs conditional on Region D being reached. We summarize this result in the following Proposition.

Proposition 5 Suppose that a price floor $\underline{b}>0$ is in place. The expected procurement cost under the English auction allowing jump-down strategy is equivalent to that under the first-price auction, i.e., $E m^{E B}=E m^{F}$.

Proof. Omitted. 
We illustrate the result with Example 1 and Example 2. In Example 1, sellers adopt the following jump-down strategy $\beta\left(c_{i}\right)=2 / 3-c_{i}$ whenever $c_{i}<1 / 3$. As such, nobody jumps down at the outset. In this case, the seller with the lower cost always wins and the auctioneer's expected cost is 0.667 , the same as that under the first-price auction. In Example 2, the seller jumps down at the outset if $c_{i}<1 / 3$, and waits to jump down until the price reaches $\beta\left(c_{i}\right)=6 / 5-c_{i}$ if $1 / 3 \leq c_{i}<3 / 5$. The auctioneer's expected cost equals 0.679 , the same as that under the first-price auction.

Finally, we want to discuss about the Dutch auction. Since the Dutch auction is strategically equivalent to the first-price auction. ${ }^{11}$ Compared with the first-price auction, the only additional information provided by the "open" mechanism of the Dutch auction is that people can observe some bidders (or bidders) has agreed to supply the item; but that causes the ending of the auction. In this sense, the information structure of the Dutch auction is the same with that of the first-price auction. Therefore, Dutch auction will perform exactly the same as the first-price auction studied here, even though the new feature of bid floor is added. To conclude, we have obtained the following ranking: as long as $\underline{b}>0, E m^{F}=E m^{D}=E m^{E B}<$ $E m^{E A}=E m^{S}$.

\section{Expected procurement costs: with versus without bid floor}

Thus far we have examined the expected costs across different auction formats when the bid floor is in place, we now examine the expected costs with versus without the bid floor. To this end, it suffices to compare $E m^{F}$ with $E m^{\widehat{F}}$. Clearly, they are equal when the bid floor under $\Gamma^{F}$ is non-binding; the comparison is less straightforward when it is binding. On the one hand, seller $i$ 's bid is pushed up if $c_{i}<c_{0}$, leading to an increase in the expected cost; on the other hand, her bid is actually reduced if $c_{i} \in\left[c_{0}, c^{*}\right]$, leading to a reduction of the expected cost. The former effect turns out to be predominant, and the expected cost is always increased when the bid floor under $\Gamma^{F}$ is binding. This can be best understood using the direct mechanism approach.

\footnotetext{
${ }^{11}$ According to Krishna (2002, p 4), "two games are strategically equivalent if they have the same normal form except for duplicate strategies. Roughly this means that for every strategy in one game, a player has a strategy in the other game, which results in the same outcomes".
} 
Under $\Gamma^{t}$, the equilibrium is characterized by an allocating rule $\mathbf{Q}^{t} \equiv\left(Q_{1}^{t}, \ldots, Q_{N}^{t}\right)$ and a payment rule $\mathbf{M}^{t} \equiv\left(M_{1}^{t}, \ldots, M_{N}^{t}\right), t=F, \widehat{F}$. Given the cost profile of all bidders, $\mathbf{c}=\left(c_{1}, \ldots, c_{N}\right), Q_{i}^{t}(\mathbf{c})$ is the probability that seller $i$ will sell its good and $M_{i}^{t}(\mathbf{c})$ $=Q_{i}^{t}(\mathbf{c}) B_{i}^{t}\left(c_{i}\right)$ is seller $i$ 's expected payment received, where $B_{i}^{t}\left(c_{i}\right)$ has been defined in (1) and (3), respectively. Seller $i$ 's expected payoff is denoted by $u_{i}^{t}\left(c_{i}\right)$, where her cost is $c_{i}$. It is straightforward to verify that $u_{i}^{t}(\bar{c})$ simply equals zero; that is, the least competitive seller is expected to earn a zero expected payoff.

By the revelation principle, this equilibrium can be equivalently represented by an equilibrium in a direct mechanism, in which sellers are asked to reveal their costs. A powerful result of this alternative formulation is that the expected cost of the auctioneer-buyer can be represented in terms of $\mathbf{Q}^{t}$ and $\mathbf{M}^{t}$ as follows, ${ }^{12}$

$$
E m^{t}=\int_{\mathbf{c} \in[0, \bar{c}]^{N}}\left[\sum_{i} \Phi\left(c_{i}\right) Q_{i}^{t}(\mathbf{c})\right] h(\mathbf{c}) d \mathbf{c}+\sum_{i} u_{i}^{t}(\bar{c})
$$

where $\Phi\left(c_{i}\right)=c_{i}+H\left(c_{i}\right) / h\left(c_{i}\right)$, which can be interpreted as the virtual cost, and $U_{i}(\bar{c})$, as argued shortly before, equals zero. We assume that the virtual cost $\Phi\left(c_{i}\right)$ is decreasing in $c_{i}{ }^{13}$ The bracket term inside the integrand has an intuitive interpretation: it is a weighted average of the virtual costs with $Q_{i}^{t}(\mathbf{c}), i=1, \ldots, N$ being the weights. Notice that in both $\mathbf{Q}^{\widehat{F}}$ and $\mathbf{Q}^{F}$ the buyer always succeeds in buying a good, i.e., $\sum_{i} Q_{i}^{t}(\mathbf{c})=1$ for all $\mathbf{c}$, and (i) under $\mathbf{Q}^{\widehat{F}}$ the buyer always buys from the least cost seller(s) but (ii) under $\mathbf{Q}^{F}$ the buyer may buy from a higher-cost seller, rather than from the least cost seller(s). As such, relative to its counterpart under $\mathbf{Q}^{F}$, the weighted average in the integrand under $\mathbf{Q}^{\widehat{F}}$ is the same for some cost profiles and is strictly smaller for other cost profiles. Then it follows that $E m^{\widehat{F}}<E m^{F}$ when the bid floor under $\Gamma^{F}$ is binding.

We then have complete comparison of expected procurement costs. In light of the comparison result obtained at the end of last section, we have the following:

- When $\underline{b}$ is non-binding in equilibrium in $\Gamma_{F}$ (also in $\Gamma_{E B}$ ), we have $E m^{j}=$ $E m^{F}=E m^{D}=E m^{E B}<E m^{E A}=E m^{S}$, where $j=\widehat{F}, \widehat{S}, \widehat{E A}, \widehat{D}$.

\footnotetext{
${ }^{12}$ In much the same way as in the forward auction where the auctioneer-seller's expected revenue can be represented by the relevant allocation rule and the payment rule. The derivation of (15) follows Chapter 5 of Krishna (2002), in which he derives the auctioneer-buyer's expected revenue under a forward selling mechanism; equation (5.12) in page 69 there is the counterpart to our equation. Also see the appendix for more discussion.

${ }^{13}$ The term $h / H$ is known as the reverse hazard rate, or Mills' rate. Thus a sufficient condition for $\Phi$ to be increasing is that the reverse hazard rate is decreasing. This sufficient condition trivially holds for uniform distributions.
} 
- When $\underline{b}$ is binding in equilibrium in $\Gamma_{F}$ (also in $\Gamma_{E B}$ ), we have $E m^{j}<E m^{F}=$ $E m^{D}=E m^{E B}<E m^{E A}=E m^{S}$, where $j=\widehat{F}, \widehat{S}, \widehat{E A}, \widehat{D}$.

We have not dealt with the question of optimal auction, but the reader can learn more about this in the appendix.

\section{Concluding remarks}

In this paper, we have assumed that an explicitly, externally given price floor is in place in a competitive bidding. This price floor is motivated by minimum wage legislation that is commonplace in all advanced industrial economies, and also by the urge of NGOs and organized labor to safeguard labor standard. We have found that given the same price floor, different auction formats would lead to different expected procurement costs on the part of the monopsony-buyer. Our research thus allows us to assess which auction format should be adopted if a bid price is to be imposed.

The equivalence of the first-price auction and the English auction with the BuyIt-Now clause is quite intriguing. We find that a seller who will bid at the price floor in the former auction if, and only if, she will jump down to sell at a posted price at the beginning in the latter auction, leading to payoff equivalence between the two auctions for all agents. This paper can be viewed as one comparing the expected revenue under different forward auctions in which there is a common upper bound to bid values, motivated by online auctions with the Buy-It-Now clause.

Two comments are in order. First, so far we study the impact of an explicit price floor on the monopsony-buyer. But such a price floor has impact to other parties as well. Whether such a bid price is desirable from the societal point of view, the cost inflicted on the buyer has to compared with the benefits brought to these other parties. Second and related, we have assumed that all bidders produce the same quality of goods and that there is commitment so that there is no re-negotiation subsequent to the auction. Both assumptions are made in order to address whether there is cost equivalence between different formats of auction. But neither of them is necessarily guaranteed in real world problems, and our analysis only serves as an intermediate step for further research. 


\section{References}

[1] Alexandersson, Gunnar and Staffan Hulten (2006), "Predatory bidding in competitive tenders: A Swedish case study," European Journal of Law and Economics 22: 73-94.

[2] Avery, Christopher (1998), "Strategic Jump Bidding in English Auctions," The Review of Economic Studies, 65: 185-210

[3] Banerjee, Priyodorshi and Archishman Chakraborty (2005), "Auctions with Ceilings," mimeo, SSRN: http://ssrn.com/abstract $=617550$

[4] Budish,-Eric-B; Takeyama,-Lisa-N. (2001), "Buy Prices in Online Auctions: Irrationality on the Internet?" Economics Letters, 72(3): 325-33.

[5] Calveras, Aleix, Juan-Jose Ganuza and Ester Hauk (2004), "Wild Bids, Gambling for Resurrection in Procurement Contracts," Journal of Regulatory Economics 26(1): 41-68.

[6] Che, Yeon-Koo and Ian Gale (1998a), "Caps on Political Lobbying," American Economic Review, 88: 643-51

[7] Che, Yeon-Koo and Ian Gale (1998b), "Standard Auctions with Financially Constrained Bidders," The Review of Economic Studies: 65: 1-21

[8] Chiu, Y. Stephen and Francis Cheung (2004), "Posted Price Clauses in Ascending Auctions," mimeo, University of Hong Kong.

[9] DGIII Working Group on Abnormally Low Tenders (1999) 'Prevention, Detection and Elimination of Abnormally Low Tenders in the European Construction Industry," http://ec.eurpoa.eu/enterprise/construction/alo/altfin.htm

[10] Gavious, A., B. Moldovanu and A. Sela (2002), "Bid Costs and Endogenous Bid Caps," RAND Journal of Economics, 33: 709-722

[11] Hidvegi, Zoltan, Wenli Wang and Andrew B. Whinston. (2006), "Buy-price English auction," Journal of Economic Theory 129: 31-56.

[12] Krishna, V. (2002), Auction Theory, Academic Press, San Diego.

[13] Laffont, Jean-Jacques and Jacques Robert (1996), "Optimal Auction with Financially Constrained Buyers." Economics Letters, 52: 181-186. 
[14] Manelli, Alejandro and Daniel Vincent (1995), "Optimal Procurement Mechanisms," Econometrica, Vol. 63: 591-620.

[15] Maskin, Eric S. (2000), "Auctions, development and privatization: efficient auctions with liquidity-constrained buyers." European Economic Review, 44: $667-681$.

[16] Maskin, Eric S. and John G. Riley (1985), "Auction theory with private values." American Economic Review Papers and Proceedings, 75: 150-155.

[17] Mathew, T (2004), "The Impact of Discounting on an Auction with a Buyout Option: a Theoretical Analysis Motivated by eBay's Buy-It-Now Feature," Journal of Economics, 81, 25-52

[18] McAfee, R. P. and J. McMillan (1987), "Auctions with a Stochastic Number of Bidders," Journal of Economic Theory, 43: 1-19

[19] Myerson, R. (1981), "Optimal Auction Design," Mathematics of Operations Research, 6: 8-73

[20] Vickrey, W. (1961), "Counterspeculation, Auctions, and Competitive Sealed Tenders", Journal of Finance, 16: 8-37

[21] Reynolds, Stanley S. and John Wooders (2009), "Auctions with a buy price," Economic Theory 38: 9-39.

[22] Rhodes-Kropf, Matthew and S. Viswanathan (2005), "Financing auction bids." RAND Journal of Economics, 36, Iss. 4, 789-815

[23] Riley, John G. (1989), "Expected revenue from open and sealed bid auctions." Journal of Economic Perspectives, 3 (3), 41-50.

[24] Riley, J. and W. Samuelson (1981), "Optimal Auctions," American Economic Review, 71: 381-392

[25] Roth, A., and A. Ockenfels (2002), "Last Minute Bidding and the Rules of Ending Second-Price Auctions: Evidence from eBay and Amazon Auctions on the Internet," American Economic Review, 92: 1093-1103

[26] Wang, R. (2000), "Bidding and Renegotiation in competitive biddings," European Economic Review, 44: 1577-1597 
[27] Zhang, Charles Z. (2001), "High Bids and Broke Winners," Journal of Economic Theory 100: 129-171. 


\section{Appendix A: Auxiliary results that help prove Proposi- tion 2}

Claim 1 Suppose $B^{F}$ is used by all sellers other than $i$. For seller $i$, regardless of $c_{i}$, bidding $B^{\widehat{F}}\left(c^{*}\right)$ is a strictly better strategy than bidding any $b \in\left(\underline{b}, B^{\widehat{F}}\left(c^{*}\right)\right)$.

Proof. By bidding $b \in\left(\underline{b}, B^{\widehat{F}}\left(c^{*}\right)\right)$ rather than $B^{\widehat{F}}\left(c^{*}\right)$, the seller wins the project with exactly the same probability but the payment received conditional on winning is strictly lower. Hence, bidding such $b$ is strictly worse.

Claim 2 Suppose $B^{F}$ is used by all sellers other than $i$. For seller $i$, bidding $\underline{b}$ is better than bidding $B^{\widehat{F}}\left(c^{*}\right)$ if and only if $c_{i}<c^{*}$.

Proof. By bidding $\underline{b}, i$ 's expected payoff is

$$
\left(\underline{b}-c_{i}\right) \times \text { probability of winning by bidding } \underline{b} \text {; }
$$

by bidding $B^{\widehat{F}}\left(c^{*}\right)$, i's expected payoff is

$$
\left(B^{\widehat{F}}\left(c^{*}\right)-c_{i}\right) \times \text { probability of winning by bidding } B^{\widehat{F}}\left(c^{*}\right) \text {. }
$$

Notice that both probabilities are independent of $c_{i}$, and both expected payoffs are linear and decreasing in $c_{i}$. It is easy to show that the former cuts the latter from above when $c_{i}=c^{*}$. The Claim is thus immediate.

Claim 3 Given that $B^{F}$ is used by all sellers other than $i$. For seller $i$, if $c_{i}<c^{*}$, then bidding $B^{\widehat{F}}\left(c^{*}\right)$ is better than bidding any $b>B^{\widehat{F}}\left(c^{*}\right)$.

Proof. We define $U_{i}^{t}\left(c_{i}, b ; B\right)$ as the expected utility for the seller $i$ with the cost of $c_{i}$ in auction game $\Gamma_{t}$, when she bids $b$ and and all other sellers adopt strategy $B$. For $b \geq B^{\widehat{F}}\left(c^{*}\right)$, we define $c_{b}$ in the equation $B^{\widehat{F}}\left(c_{b}\right)=b$. Moreover, since $B^{\widehat{F}}$ and $B^{F}$ coincide for $c \geq c^{*}, B^{F}\left(c_{b}\right)=B^{\widehat{F}}\left(c_{b}\right)=b$.

$$
\begin{aligned}
U_{i}^{F}\left(c_{i}, b ; B^{F}\right) & =\left(b-c_{i}\right) \operatorname{Pr}\left(\min _{j \neq i}\left\{B^{F}\left(c_{j}\right)\right\}>b\right) \\
& =\left(b-c_{i}\right) \operatorname{Pr}\left(Y_{1}>c_{b}\right) \\
& =\left[\left(b-c^{*}\right)+\left(c^{*}-c_{i}\right)\right] \operatorname{Pr}\left(Y_{1}>c_{b}\right) \\
& =\left(b-c^{*}\right) \operatorname{Pr}\left(Y_{1}>c_{b}\right)+\left(c^{*}-c_{i}\right) \operatorname{Pr}\left(Y_{1}>c_{b}\right) .
\end{aligned}
$$


Notice that the first term is weakly smaller than $\left(B^{\widehat{F}}\left(c^{*}\right)-c^{*}\right) \operatorname{Pr}\left(Y_{1}>c^{*}\right)$ (since $B^{\widehat{F}}\left(c^{*}\right)$ is a best response for seller with cost $c^{*}$ when facing all others using $\left.B^{\widehat{F}}\right)$ and the second term is weakly smaller than $\left(c^{*}-c_{i}\right) \operatorname{Pr}\left(Y_{1}>c^{*}\right)$ (since $\operatorname{Pr}\left(Y_{1}>c\right)$ is decreasing in $c$ ). Therefore,

$$
\begin{aligned}
U_{i}^{F}\left(c_{i}, b ; B^{F}\right) & \leq\left(B^{\widehat{F}}\left(c^{*}\right)-c^{*}\right) \operatorname{Pr}\left(Y_{1}>c^{*}\right)+\left(c^{*}-c_{i}\right) \operatorname{Pr}\left(Y_{1}>c^{*}\right) \\
& =\left(B^{\widehat{F}}\left(c^{*}\right)-c_{i}\right) \operatorname{Pr}\left(Y_{1}>c^{*}\right) \\
& =\left(B^{\widehat{F}}\left(c^{*}\right)-c_{i}\right) \operatorname{Pr}\left(\min _{j \neq i}\left\{B^{\widehat{F}}\left(c_{j}\right)\right\}>B^{\widehat{F}}\left(c^{*}\right)\right) \\
& =\left(B^{\widehat{F}}\left(c^{*}\right)-c_{i}\right) \operatorname{Pr}\left(\min _{j \neq i}\left\{B^{F}\left(c_{j}\right)\right\}>B^{\widehat{F}}\left(c^{*}\right)\right) \quad\left(B^{\widehat{F}} \text { and } B^{F} \text { coincide for } c \geq c^{*}\right) \\
& =U_{i}^{F}\left(c_{i}, B^{\widehat{F}}\left(c^{*}\right) ; B^{F}\right) .
\end{aligned}
$$

The proof is complete.

Claim 4 Suppose $B^{F}$ is used by all sellers other than $i$. For seller $i$, if $c_{i} \geq c^{*}$, then bidding $B^{\widehat{F}}\left(c_{i}\right)$ is better than bidding any different $b \geq B^{\widehat{F}}\left(c^{*}\right)$.

Proof. We define $U_{i}^{t}\left(c_{i}, b ; B\right)$ in the same fashion as in the proof of Claim 3. Since $B^{\widehat{F}}$ constitutes a Bayesian Nash equilibrium in the unrestricted game $\Gamma_{\widehat{F}}$, we have

$$
U_{i}^{\widehat{F}}\left(c_{i}, B^{\widehat{F}}\left(c_{i}\right) ; B^{\widehat{F}}\right) \geq U_{i}^{\widehat{F}}\left(c_{i}, b ; B^{\widehat{F}}\right) \text { for all } b \neq B^{\widehat{F}}\left(c_{i}\right) .
$$

We first notice that, for $c_{i} \geq c^{*}$,

$$
U_{i}^{F}\left(c_{i}, B^{\widehat{F}}\left(c_{i}\right) ; B^{F}\right)=U_{i}^{\widehat{F}}\left(c_{i}, B^{\widehat{F}}\left(c_{i}\right) ; B^{\widehat{F}}\right)
$$

The reason is that, in each case, the payoff conditional on winning the project is the same $\left(B^{\widehat{F}}\left(c_{i}\right)-c_{i}\right)$ and that the probability of winning is also the same $\left(\operatorname{Pr}\left(Y_{1}>c_{i}\right)\right)$. We also notice that, for $c_{i} \geq c^{*}$,

$$
U_{i}^{F}\left(c_{i}, b ; B^{F}\right)=U_{i}^{\widehat{F}}\left(c_{i}, b ; B^{\widehat{F}}\right) \text { for all } b \neq B^{\widehat{F}}\left(c_{i}\right) \text { and } b \geq B^{\widehat{F}}\left(c^{*}\right) .
$$

The reason is that, in each case, the payoff conditional on winning the project is the same $\left(b-c_{i}\right)$ and that the probability of winning is also the same. (Notice that the probabilities of winning are $\operatorname{Pr}\left(\min _{j \neq i}\left\{B^{F}\left(c_{j}\right)\right\}>b\right)$ and $\operatorname{Pr}\left(\min _{j \neq i}\left\{B^{\widehat{F}}\left(c_{j}\right)\right\}>b\right)$, 
respectively, and since $B^{F}$ and $B^{\widehat{F}}$ coincide for $c_{j} \geq c^{*}$, the two probabilities are indeed the same.) Therefore, using (16), we have, for $c_{i} \geq c^{*}$,

$$
U_{i}^{F}\left(c_{i}, B^{\widehat{F}}\left(c_{i}\right) ; B^{F}\right) \geq U_{i}^{F}\left(c_{i}, b ; B^{F}\right) \text { for all } b \neq B^{\widehat{F}}\left(c_{i}\right) \text { and } b \geq B^{\widehat{F}}\left(c^{*}\right)
$$

The proof is completed.

\section{Appendix B: Proofs of Propositions and Lemmas stated in the main text \\ Proof of Proposition 2}

Proof. The proof to part (a) is straightforward and is omitted.

For part (b), given that every other seller adopts $B^{F}$, we now check seller $i$ 's incentive. If $c_{i}<c^{*}$, then Claims 1, 2, and 3 (in Appendix A) imply that the optimal response is to bid $\underline{b}$; if $c_{i}>c^{*}$, Claims 1,2 , and 4 (in Appendix A) imply that the optimal response is to bid $B^{F}\left(c_{i}\right)=B^{\widehat{F}}\left(c_{i}\right)$; if $c_{i}=c^{*}$, Claims 1 and 4 imply that both bidding $\underline{b}$ and bidding $B^{\widehat{F}}\left(c^{*}\right)$ are best responses.

\section{Proof of Proposition 3}

Proof. For sellers with $c_{i} \in[\underline{b}, \bar{c}]$, staying active until price drops to $c_{i}$ is her weakly dominant strategy. For sellers with $c_{i} \in[0, \underline{b})$, they will jump down to bid $\underline{b}$ at a price of $\beta\left(c_{i}\right)$. Given that all other sellers adopt this jump-down strategy $\beta($.$) ,$ bidder $i$ with cost $c_{i}$ chooses $\mathrm{b}$ in the following program:

$$
\max _{b} \int_{\beta^{-1}(b)}^{b}\left(\underline{b}-c_{i}\right) d G(y)+\int_{b}^{\bar{c}}\left(y-c_{i}\right) d G(y) .
$$

Differentiating it with respect to $b$ and imposing the symmetry condition that $\beta\left(c_{i}\right)=b$, we obtain

$$
g\left(c_{i}\right) c_{i}-g\left(\beta\left(c_{i}\right)\right) \beta\left(c_{i}\right) \frac{d \beta\left(c_{i}\right)}{d c_{i}}=\underline{b}\left(g\left(c_{i}\right)-g\left(\beta\left(c_{i}\right)\right) \frac{d \beta\left(c_{i}\right)}{d c_{i}}\right) .
$$

By rearranging terms and integrating both sides for a range of $c_{i}$ in which the equation holds true, we obtain

$$
\int_{c_{i}}^{\beta\left(c_{i}\right)} y d G(y)=\underline{b} \int_{c_{i}}^{\beta\left(c_{i}\right)} d G(y),
$$

which is equivalent to

$$
E\left[Y_{1} \mid c_{i}<Y_{1}<\beta\left(c_{i}\right)\right]=\underline{b}
$$


If $\beta(\underline{c}) \leq \bar{c}$, any seller with $c_{i} \in[0, \underline{b})$ stays active until price drops to $\beta\left(c_{i}\right)$ at which she will jump to bid $\underline{b}$. If $\beta(\underline{c})>\bar{c}$, sellers with $c_{i} \in\left[0, c^{* *}\right]$ would bid $\underline{b}$ at the outset of the auction. The existence of $c^{* *}$ can be proved in the same way with the existence of $c^{*}$, so it is omitted. In this case, like the equilibrium bidding strategy in $\Gamma_{F}$, the bidding strategy in $\Gamma_{E B}$ also exhibits a discontinuity at a critical point $c^{* *}$. The proof is basically similar and hence omitted.

\section{Appendix C: Additional discussion related to Section 7}

Instead of studying $\Gamma^{F}$ and $\Gamma^{\widehat{F}}$, we simply look at any incentive compatible direct mechanism $(\mathbf{Q}, \mathbf{M})$, where $\mathbf{Q}$ and $\mathbf{M}$ are the corresponding allocating rule and payment rule. In this case, the auctioneer-buyer's problem should be to maximize his expected payoff, which equals expected gross benefit minus expected cost, i.e.,

$$
\int_{c \in[0, \bar{c}]^{N}}\left[\sum_{i}\left(R-\Phi\left(c_{i}\right)\right) Q_{i}(\mathbf{c})\right] h(\mathbf{c}) d \mathbf{c}+\sum_{i}\left(-u_{i}(\bar{c})\right)
$$

where $R$ is the buyer's utility from obtaining the good and $R-\Phi$ can be interpreted as the virtual valuation. The bracket term inside the integrand has an intuitive interpretation: it is a weighted average of the virtual valuations with $Q_{i}(\mathbf{c}), i=1, \ldots, N$ being the weights. The optimal mechanism requires that the auctioneer-buyer buys from any seller whose corresponding virtual valuation is both non-negative and the greatest among all sellers. Under the assumption that the virtual valuation is decreasing in $c_{i}$, the optimal mechanism can be in the form of a second-price sealed bid

auction with a reserve price $p^{*}$ above which the buyer will not buy, where $p^{*}$ satisfies $R-\Phi\left(p^{*}\right)=0$. The virtual valuation, as well as the optimal reserve price, can be understood as follows. Suppose the monopsony-buyer is facing a single seller, making a take-it-or-leave-it offer $p$. Then the probability that the seller will accept the offer is $H(p)$, the probability her cost is smaller than $p$. Then the buyer's expected payoff is $H(p) B-H(p) p$, where $H(p) R$ is his total benefit and $p H(p)$ total cost. Differentiating the expected payoff with respect to $p$ yields the first order condition $h(p)(B-p)-H(p)=0$, which with some manipulation becomes $R-\Phi(p)=0$. The optimal TIOLI price $p^{*}$ is the one that satisfies the condition. Suppose $R=1.5 \bar{c}$ and the cost is uniformly distributed in $[0, \bar{c}]$. Then the TIOLI price equals $0.75 \bar{c}$, which is also the optimal reserve price in a second-price sealed bid (reverse) auction. 